Surveys in Differential Geometry XIV

\title{
Brill-Noether theory
}

\author{
Joe Harris
}

\section{Introduction}

In this paper I'll try to give an overview of one of the central threads in the study of algebraic curves: Brill-Noether theory. After an initial discussion in Section 1 of what I mean by Brill-Noether theory, and a discussion of the role it plays in our understanding of algebraic curves, I'll give in Section 2 a summary of what is known. Finally, Sections 3 and 4 represent the real point of the paper: in these, I'll give two possible answers to the question, "where do we go from here?"

\section{What do we mean by Brill-Noether theory?}

We should start by saying what we mean by "Brill-Noether theory," and how we see its role in the larger framework of the theory of algebraic curves.

By way of analogy, consider the development of group theory. In its early days, in the $19^{\text {th }}$ century, a group was taken to be a subset of $G L_{n}$ closed under matrix multiplication and inversion - in other words, what we would now call the data of an abstract group $G$ together with a faithful representation $G \hookrightarrow G L_{n}$ of $G$. In the $20^{\text {th }}$ century, the notion of an abstract group was introduced, which had the effect of breaking what had been the study of group theory into two complementary parts. First there was the analysis of the structure of abstract groups, hopefully leading to their classification. Secondly, there was representation theory: the study, for a given abstract group $G$, of the ways in which $G$ could be realized as a subgroup of $G L_{n}$ (or, more generally, mapped to $G L_{n}$ ). This bifurcation of the subject (which applied in various contexts: finite groups, Lie groups, etc.) lent tremendous clarity and power to the study of groups.

Very much the same transformation occurred, during much the same period, in the subject of algebraic geometry. To focus on the case of algebraic curves, in the earliest days of the subject a curve meant simply an irreducible polynomial in two variables, or rather an equivalence class of such under an equivalence relation amounting to birational isomorphism. In the latter

(C)2009 International Press 
$19^{\text {th }}$ century, curves in higher-dimensional space were also considered, but one way or another a "curve" always meant a subset of projective space defined by polynomial equations. In particular, to a $19^{\text {th }}$ century geometer the problem, "classify all algebraic curves" would have meant "classify all such subsets of projective space" - in modern language, "describe the components of the Hilbert scheme $\mathcal{H}$ whose general point corresponds to an integral curve."

Then in the $20^{\text {th }}$ century the notion of an abstract curve took hold, and our view of the subject underwent a fundamental shift. Now, if you ask a geometer to "classify all curves," the problem would most likely be interpreted as meaning, "describe the moduli spaces $M_{g} . "$ By the same token, the classical problem of classifying curves in projective space can be thought of as having two distinct components: one, to describe the set of all abstract curves; and two, for a given abstract curve $C$, to describe all the ways in which $C$ may be mapped to projective space $\mathbb{P}^{r}$. Brill-Noether theory is the study of the second of these problems - in brief, it's representation theory for curves.

The analogy with group theory is, I think, an apt one. Many subjects in mathematics underwent a similar transformation between the $19^{\text {th }}$ and $20^{\text {th }}$ century, in which the fundamental objects of study went from subobjects of a standard ambient object to ones defined abstractly as sets with additional structure (think of what "differentiable manifold" means to us today, and what it meant a century ago). There is, however, one fundamental respect in which algebraic geometry differs from many other disciplines: the objects we study - varieties, and more generally schemes - fall naturally into families that are themselves parametrized by other schemes, called moduli or parameter spaces. Thus, the set of all smooth, complete curves of genus $g$ has naturally the structure of a variety, the moduli space $M_{g}$; and, for a given curve $C$, the set of all nondegenerate maps $C \rightarrow \mathbb{P}^{r}$ of degree $d$ (up to projective equivalence) has the structure of a scheme, denoted $\tilde{G}_{d}^{r}(C)$ (about which more in a moment).

Each of these facts has an impact on what we mean by "Brill-Noether theory." To begin with, the fact that curves of a given genus are parametrized by a variety means that the problem "describe all the ways in which an arbitrary curve $C$ may be mapped to projective space $\mathbb{P}^{r}$ " admits an important subproblem: to describe such maps for a general curve $C$ of genus $g$. And, while there are many theorems concerning maps of an arbitrary curve to projective space (Clifford's theorem, Martens' theorem, Castelnuovo's bound and many variants and extensions of these), traditional Brill-Noether theory has focussed on the problem for general curves.

Secondly, the fact that the set of maps of a given curve to projective space has the structure of a variety means that we can ask many more questions about the geometry of this variety: not just whether it's empty or not, but what its dimension is; whether it's irreducible or not; if it's smooth, and, if not, what its singular locus is, and so on. All these questions are usually viewed as in the purview of Brill-Noether theory. 


\section{Classical Brill-Noether theory}

I'd like to describe here the main results of Brill-Noether theory, at least up to the 1980s. ("Classical" is a relative term.) To begin with, let's establish some background and ground rules. Throughout, $C$ will denote a smooth, complete curve of genus $g$. It is of some interest to extend our inquiry to singular curves as well - if nothing else, they appear naturally in the proofs of many of the standard results - but since much of what we can say about maps on singular curves derives from studying their normalizations, we will leave these aside for now.

To begin with, we'll restrict our attention to nondegenerate maps, that is, maps $f: C \rightarrow \mathbb{P}^{r}$ whose image does not lie in a hyperplane. This avoids redundancy: a degenerate map is best thought of as a nondegenerate map $C \rightarrow \mathbb{P}^{s}$ for some $s<r$ composed with a linear inclusion $\mathbb{P}^{s} \rightarrow \mathbb{P}^{r}$.

By the degree of a map $f: C \rightarrow \mathbb{P}^{r}$ we mean the degree of the pullback divisor $f^{*} H$ for any hyperplane $H \subset \mathbb{P}^{r}$-in other words, the degree of the image $f(C) \subset \mathbb{P}^{r}$ times the degree of the map $C \rightarrow f(C)$.

Now, a nondegenerate map $C \rightarrow \mathbb{P}^{r}$ of degree $d$, modulo automorphisms of $\mathbb{P}^{r}$, corresponds to the following data:

- a line bundle $L$ of degree $d$ on $C$; and

- an $(r+1)$-dimensional vector space $V \subset H^{0}(L)$ of sections of $L$ such that

- the sections $\{\sigma \in V\}$ have no common zeroes.

The pair $(L, V)$ is called a linear system of degree $d$ and dimension $r$ on $C$, and is usually referred to as a $g_{d}^{r}$ for short. The last condition is expressed by saying that the linear system is base point free. And here is where we deviate from our stated purpose for the first time: while the set of base point free $g_{d}^{r}$ s on a curve $C$ is indeed parametrized by a variety, as we said earlier, that variety will not in general be complete: a linear system without base points may well specialize to one with them. Since so many of our techniques apply primarily to complete varieties, we will usually drop the condition that our linear system be base point free, and study instead the variety $G_{d}^{r}(C)$ parametrizing all linear systems of degree $d$ and dimension $r$ on $C$. This variety, it turns out, is in fact projective.

Note that this is a relatively harmless deviation: a linear system $(L, V)$ with a base point $p \in C$, for example, may be viewed as a linear system $\left(L^{\prime}, V^{\prime}\right)$ with $L^{\prime}=L(-p)$ and $V$ the image of $V^{\prime}$ under the sheaf map $L^{\prime} \rightarrow L$ vanishing at $p$. Any linear system can in this way be realized uniquely as a base point free linear system of lower degree via such an inclusion; in other words, if we can describe the base point free linear systems of degree $e<d$, we can describe the set of linear systems of degree $d$ with base points and hence its complement in $G_{d}^{r}(C)$.

For a detailed description of how the schemes $G_{d}^{r}(C)$ may be defined and constructed, see $[\mathbf{A C G H}]$. We may also define analogously a scheme $\mathcal{G}_{d}^{r}$ parametrizing triples $(C, L, V)$ consisting of a smooth curve $C$, a line bundle 
$L$ of degree $d$ on $C$ and an $(r+1)$-dimensional vector space of sections of $L$; naturally, $\mathcal{G}_{d}^{r}$ admits a map to the moduli space $M_{g}$ of smooth curves, with fiber $G_{d}^{r}(C)$ over the point $[\mathbf{C}] \in M_{g}$. Finally, we define the Brilll-Noether number $\rho(g, r, d)$ by

$$
\rho(g, r, d)=g-(r+1)(g-d+r) .
$$

With these conventions, the basic results of Brill-Noether theory fall roughly into three categories. (Rather than try to list all the original sources, we refer the reader to $[\mathbf{A C G H}]$ and $[\mathbf{H M}]$, which contain complete statements of these results and all relevant references.)

\section{Existence and nonexistence results:}

a. When $\rho \geq 0$, every curve of genus $g$ possesses a $g_{d}^{r}$; that is, $G_{d}^{r}(C) \neq \emptyset$ for any $C$ ([ACGH V.1.1]); and conversely

b. When $\rho<0$, a general curve does not possess a $g_{d}^{r}$ ([HM p.261-2]).

There are immediate refinements of this statement that take into account ramification at specified points. Briefly: if $V \subset H^{0}(L)$ is a $g_{d}^{r}$ on a curve $C$ and $p \in C$ any point, we can write the orders of vanishing at $p$ of sections $\sigma \in V$ as

$$
\left\{\operatorname{ord}_{\mathrm{p}}(\sigma)\right\}_{\sigma \in V}=\left\{a_{0}, a_{1}, \ldots, a_{r}\right\}
$$

with $a_{0}<a_{1}<\cdots<a_{r}$. The associated non-decreasing sequence $\alpha_{0}, \ldots, \alpha_{r}$ defined by $\alpha_{i}=a_{i}-i$ is called the ramification sequence of the linear system $(L, V)$ at $p$, and the sum $\alpha(V, p)=\sum \alpha_{i}$ the total ramification index. If $\left(C ; p_{1}, \ldots, p_{n}\right)$ is an $n$-pointed curve and $(L, V)$ a $g_{d}^{r}$ on $C$, we define the adjusted Brill-Noether number of $(L, V)$ on $\left(C ; p_{1}, \ldots, p_{n}\right)$ to be

$$
\tilde{\rho}=\rho(g, r, d)-\sum \alpha\left(V, p_{i}\right) .
$$

Statement (b) above may then be extended to

$\mathrm{b}^{\prime}$. If $\left(C, p_{1}, \ldots, p_{n}\right)$ is a general $n$-pointed curve of genus $g$ and $(L, V)$ any $g_{d}^{r}$ on $C$, then the adjusted Brill-Noether number $\tilde{\rho} \geq 0$ ([HM 5.37]).

The existence statement (a) is trickier. The correct refinement (which subsumes $\left.\left(b^{\prime}\right)\right)$ is

$\mathrm{a}^{\prime}$ : If $\left(C, p_{1}, \ldots, p_{n}\right)$ is a general $n$-pointed curve of genus $g$ and $\alpha^{j}=$ $\left(\alpha_{0}^{j}, \ldots, \alpha_{r}^{j}\right)$ for $j=1, \ldots, n$ any collection of nondecreasing sequences, there exists a $g_{d}^{r}$ on $C$ with

$$
\alpha_{i}\left(V, p_{j}\right) \geq \alpha_{i}^{j} \quad \forall i, j
$$

if and only the product of Schubert classes

$$
\left(\sigma_{r}\right)^{g} \cup \prod_{j} \sigma_{\alpha_{0}^{j}, \ldots, \alpha_{r}^{j}} \neq 0
$$

in the Chow ring of the Grassmannian $\mathbb{G}(d-r-1, d)$ ([HM 5.42]).

2. Results about the geometry of $G_{d}^{r}(C)$ for general $C$ :

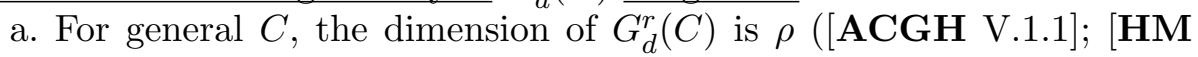
$5.37])$. 
b. For general $C$, the scheme $G_{d}^{r}(C)$ is smooth ([ACGH V.1.6]).

c. For $\rho>0$ and $C$ general, the scheme $G_{d}^{r}(C)$ is irreducible ([ACGH V.1.4]).

d. When $\rho=0$, by (2a) a general curve $C$ has a finite number of $g_{d}^{r} \mathrm{~s}$, and as $C$ varies in $M_{g}$, the monodromy action on these $g_{d}^{r}$ s is transitive -in other words, there is a unique irreducible component of $\mathcal{G}_{d}^{r}$ dominating $M_{g}$ $([\mathbf{E H}])$.

e. For general $C$, the class of the image $W_{d}^{r}(C)$ of $G_{d}^{r}(C)$ in $\operatorname{Pic}^{d}(C)$ is given ([ACGH V.1.3]) by

$$
\left[W_{d}^{r}(C)\right]=\left(\prod_{i=0}^{r} \frac{i !}{(g-d+r+i) !}\right) \theta^{(r+1)(g-d+r)} .
$$

In particular, in case $\rho=0$ the number of $g_{d}^{r}$ s on a general curve $C$ is

$$
\# W_{d}^{r}(C)=g ! \prod_{i=0}^{r} \frac{i !}{(g-d+r+i) !}
$$

3. Results about the geometry of $g_{d}^{r}$ s and the corresponding maps $C \rightarrow \mathbb{P}^{r}$ on a general curve $C$ :

a. For $C$ general and any $(L, V) \in G_{d}^{r}(C)$, the multiplication map

$$
H^{0}(L) \otimes H^{0}\left(K L^{-1}\right) \rightarrow H^{0}(K)
$$

is injective ([ACGH V.1.7], [HM 5.78]).

b. For $C$ general and any $(L, V) \in G_{d}^{r}(C)$ with $r>0$,

$$
H^{1}\left(L^{2}\right)=0
$$

([ACGH p. 126 and V.1.7]).

c. If $r \geq 3$, for general $C$ and a general point $(L, V) \in G_{d}^{r}(C)$ the map $f: C \rightarrow \mathbb{P}^{r}$ associated to the linear system $(L, V)$ is an embedding;

d. If $r=2$, for general $C$ and a general point $(L, V) \in G_{d}^{r}(C)$ the map $f: C \rightarrow \mathbb{P}^{r}$ associated to the linear system $(L, V)$ maps $C$ birationally onto a plane curve with only nodes as singularities; in particular, it's an immersion; and

e. If $r=1$, for general $C$ and a general point $(L, V) \in G_{d}^{r}(C)$ the map $f: C \rightarrow \mathbb{P}^{r}$ associated to the linear system $(L, V)$ expresses $C$ as a simply branched cover of $\mathbb{P}^{1}$.

(When $\rho=0$, statements (c), (d) and (e) above apply to all $g_{d}^{r}$ s on a general curve $C$.)

This concludes our summary of known results in Brill-Noether theory. The question I'd like to take up now is: what's next? There are two essentially independent directions for further study, roughly corresponding to the second and third categories of results above: we can ask to know more about the geometry of general curves, embedded in projective space by general linear series; and we can ask to know more about linear series on special curves. In the last two sections, I'll take up these issues in turn. 


\section{The geometry of general curves in projective space}

As we've said, Brill-Noether theory may be viewed as providing a bridge between the realms of abstract curves and curves in projective space: given a curve - or at least a general one - the theory tells us, for example, that it can be embedded as a curve of a certain degree in a certain projective space. But this is just the beginning: once a curve is embedded in projective space, there are all sorts of questions we can ask about it. Most notably, since a curve in projective space is the zero locus of polynomials, we want to know what sort of polynomials define it - how many homogeneous polynomials of each degree $m$ vanish on the curve (that is, the Hilbert function of the curve), and what is a minimal set of generators for the homogeneous ideal of the curve. And the questions continue from there: if we know generators for the ideal, we can ask how many relations among these generators there are in each degree, and for generators of the module of relations, and so on.

We don't have many answers to these questions. We do have, however, a consistent and plausible series of conjectures that, if correct, will answer all our questions, and that have been verified by Voisin $([\mathrm{V}])$ in at least one crucial case.

Rather than trying to describe these conjectures in general, we'll focus on one example; the picture in general should be clear.

The example we'll consider is this: given a general curve $C$ of genus 8 , how may $C$ be embedded in projective space? From the genus formula for plane curves, it's clear that $C$ cannot be embedded in $\mathbb{P}^{2}$, so we look next to nondegenerate maps $C \rightarrow \mathbb{P}^{3}$. Here Brill-Noether theory tells us that the minimal degree of such a map is 9 ; that there are finitely many such maps up to projective equivalence ( 42 , to be precise), and that they're all embeddings. We'll look at such an embedding, accordingly, and ask: what can we say about the equations defining the image curve $C \subset \mathbb{P}^{3}$ ?

As we indicated, the first and simplest question we might ask in this regard is: for each $m$, what is the dimension of the vector space of homogeneous polynomials of degree $m$ on $\mathbb{P}^{3}$ vanishing on $C$ ? For example, does $C$ lie on a quadric surface? On a cubic?

There is a standard first approach to this. We consider the restriction map

$$
\rho_{2}: H^{0}\left(\mathcal{O}_{\mathbb{P}^{3}}(2)\right) \rightarrow H^{0}\left(\mathcal{O}_{C}(2)\right)
$$

whose kernel is the vector space of quadrics vanishing on $C$, that is, the second graded piece of the homogeneous ideal $I(C)$ of $C$. We know the dimension of both spaces: on the left, we have simply the space of homogeneous quadrics in 4 variables, whose dimension is

$$
h^{0}\left(\mathcal{O}_{\mathbb{P}^{3}}(2)\right)=\left(\begin{array}{l}
5 \\
2
\end{array}\right)=10 ;
$$

on the right, Riemann-Roch tells us that

$$
h^{0}\left(\mathcal{O}_{C}(2)\right)=2 \cdot 9-8+1=11 \text {. }
$$


We thus have no reason to believe the map $\rho_{2}$ is not injective; and we expect, accordingly, that the curve $C$ will lie on no quadrics. (This is readily verified directly: there are no smooth curves of degree 9 and genus 8 on a quadric.)

Next, we look at cubics: again, we have the restriction map

$$
\rho_{3}: H^{0}\left(\mathcal{O}_{\mathbb{P}^{3}}(3)\right) \rightarrow H^{0}\left(\mathcal{O}_{C}(3)\right)
$$

and again we know the dimension of the spaces in question:

$$
h^{0}\left(\mathcal{O}_{\mathbb{P}^{3}}(3)\right)=\left(\begin{array}{l}
6 \\
3
\end{array}\right)=20,
$$

while

$$
h^{0}\left(\mathcal{O}_{C}(3)\right)=3 \cdot 9-8+1=20 .
$$

Again, our expectation would be that $\rho_{3}$ is injective; that is, the curve $C$ lies on no cubic surfaces. Again, this may be verified directly, though with some more effort: there do exist smooth curves of this degree and genus on cubic surfaces, but a dimension count tells us a general such curve does not.

What about quartics? We have

$$
h^{0}\left(\mathcal{O}_{\mathbb{P}} 3(4)\right)=\left(\begin{array}{l}
7 \\
3
\end{array}\right)=35,
$$

while

$$
h^{0}\left(\mathcal{O}_{C}(4)\right)=4 \cdot 9-8+1=29
$$

we conclude that the curve $C$ must lie on at least 6 independent quartics, and we might expect that it lies on exactly this many.

We can continue in this way to at least guess the dimensions of the graded pieces of the homogeneous ideal $I(C)$, based on the expectation that the restriction maps $\rho_{m}$ have maximal rank. In the specific case we're considering here we can actually verify that this is correct: for example, we see that $C$ is residual to a curve $C^{\prime}$ of degree 7 and genus 4 in the complete intersection of two of the quartics containing it, and use our knowledge of the simpler curve $C^{\prime}$ to count the surfaces containing $C$. This approach doesn't work in general - a general curve of high genus, embedded in $\mathbb{P}^{3}$, is not linked to any curves of lower degree or genus - but based on our experience in this and many other examples, we may make the

Maximal Rank Conjecture, Part 1: If $C$ is a general curve, $(L, V)$ a general $g_{d}^{r}$ on $C$ with $r \geq 3$ and $\phi: C \rightarrow \mathbb{P}^{r}$ the corresponding embedding, then the restriction map

$$
\rho_{m}: H^{0}\left(\mathcal{O}_{\mathbb{P}^{r}}(m)\right) \rightarrow H^{0}\left(\mathcal{O}_{C}(m)\right)
$$

has maximal rank - that is, is either injective or surjective - for every $m$.

Assuming this, we know the Hilbert function of $C$; for example, in the example above, we conclude that $C$ lies on six quartics, 18 quintics, 37 sextics, and so on.

This answers our first question, but not our second: we'd still like to know a minimal set of generators for the homogeneous ideal $I$ of $C$. In the 
present example, we might ask specifically: is $I$ generated in degree 4? - that is, do the six quartic polynomials vanishing on $C$ generate its homogeneous ideal?

The first thing to check is that this is possible dimensionally. For example, we've calculated (assuming the maximal rank conjecture) that $C$ lies on 18 quintics. Can these all be products of the six quartics with linear forms? The answer is yes: the vector space $I_{4} \otimes S_{1}$ has dimension $24 \geq 18$, so that the multiplication map

$$
I_{4} \otimes S_{1} \rightarrow I_{5}
$$

will be surjective unless there are seven or more linear relations among the six quartics, which we have no reason to expect. In the same way, we have

$$
\operatorname{dim}\left(I_{4} \otimes S_{2}\right)=60
$$

while

$$
\operatorname{dim} I_{6}=37
$$

so we might likewise guess that the multiplication map

$$
I_{4} \otimes S_{2} \rightarrow I_{6}
$$

is surjective. Continuing in this way, we are led to guess that the six quartics vanishing on $C$ do indeed generate its homogeneous ideal; and once more this can be verified directly.

Another example would be instructive here. Suppose now that $C$ is a general curve of genus 9. Brill-Noether theory tells us that $C$ may be embedded in $\mathbb{P}^{3}$ as a curve of degree 10; we'd like to describe the ideal $I$ of $C$ under a general such embedding. We start by determining the dimensions of the graded pieces of $I$, assuming Part I of the maximal rank conjecture: since $\operatorname{dim} S_{m}=\left(\begin{array}{c}m+3 \\ 3\end{array}\right)$ while $h^{0}\left(\mathcal{O}_{C}(m)\right)=10 m-8$ for all $m \geq 2$, we expect that $C$ will lie on no quadrics or cubics, while

$$
\begin{aligned}
& \operatorname{dim} I_{4}=35-32=3 ; \\
& \operatorname{dim} I_{5}=56-42=14 ; \\
& \operatorname{dim} I_{6}=84-52=32 ; \\
& \operatorname{dim} I_{7}=120-62=58
\end{aligned}
$$

and so on. Now, from the first we see that $I$ has three generators $F_{1}, F_{2}$ and $F_{3}$ in degree 4 . Moreover, from the inequality $4 \times 3 \leq 14$, we might guess that the multiplication map

$$
I_{4} \otimes S_{1} \rightarrow I_{5}
$$

is injective - in other words, these three satisfy no linear relations. This in turn means that $I$ will need an additional $14-12=2$ generators $G_{1}$ and $G_{2}$ in degree 5 .

Are we done? Do the five polynomials $F_{i}$ and $G_{i}$ generate $I$ ? Well, in degree 6 we have $3 \times 10=30$ products of the form $F_{i} \cdot Q$, with $Q$ a quadric, and $2 \times 4=8$ products of the form $G_{i} \cdot L$ with $L$ linear. Since the actual 
dimension of $I_{6}$ is $32 \leq 30+8$, we might expect that the $F_{i}$ and $G_{i}$ generate, in degree 6 at least. Similarly, in degree 7 we have

$$
3 \times 20+2 \times 10=80 \geq 58,
$$

so we might expect that they generate in degree 7 . The same calculation in general degree leads us to conjecture that $F_{1}, F_{2}, F_{3}, G_{1}$ and $G_{2}$ are indeed a minimal set of generators for $I$.

Thus we are led to append a further statement to the maximal rank conjecture. The MRC as stated above, applied to a general curve $C \subset \mathbb{P}^{r}$ by which we mean a general abstract curve $C$, embedded in $\mathbb{P}^{r}$ by a general $g_{d}^{r}$ - tells us the Hilbert function of $C$. We now go further and make the

Maximal Rank Conjecture, Part 2: The polynomials vanishing on $C$ satisfy no more relations than they are forced to, given the Hilbert function of $C$.

Given this, we can as in the examples above determine the degrees of a minimal set of generators of the homogeneous ideal $I$ of $C$.

Of course, it doesn't stop here. Given a minimal set of generators for the ideal $I$ of our general curve, we naturally would like to describe all the relations among these generators: in other words, if $I$ has $a_{d}$ generators in degree $d$, we have a surjection of modules over the polynomial ring $S$ of $\mathbb{P}^{r}$

$$
\oplus S(-d)^{\oplus a_{d}} \rightarrow I
$$

if we let $M$ be the kernel of this map - the module of relations - we'd like to describe a minimal set of generators for $M$ in turn. Again, we know (conjecturally) the Hilbert function of $M$; and if we further conjecture that the multiplication maps

$$
M_{k} \otimes S_{\ell} \rightarrow M_{\ell}
$$

again have maximal rank, this determines the degrees of a minimal set of generators for $M$. If we have $b_{e}$ generators in degree $e$, this gives us a map

$$
\oplus S(-e)^{\oplus b_{e}} \rightarrow M
$$

or in other words a three-term exact sequence

$$
\oplus S(-e)^{\oplus b_{e}} \rightarrow \oplus S(-d)^{\oplus a_{d}} \rightarrow I .
$$

If we let $N$ be the kernel of this map in turn, we know the Hilbert function of $N$, and hence - once more conjecturing that the multiplication maps have maximal possible rank - a minimal set of generators for it. Continuing in this way, we can at least guess at all the terms in a minimal resolution of the ideal $I$ of $C$.

But by now we have gone far beyond what is reasonable or true. There are currently no known counterexamples to the two Maximal Rank Conjectures above, but Eisenbud and Schreyer have exhibited examples of general linear series for which the multiplication maps on the module of relations fail to have maximal rank $([\mathbf{E S}])$. As for the conjectures above, we can verify them in relatively low-degree individual cases, when the curve $C \subset \mathbb{P}^{r}$ lies 
on surfaces whose geometry we understand (scrolls, del Pezzo surfaces, etc.). The original MRC (Part I above) was proved by Ballico and Ellia $[\mathbf{B E}]$ for nonspecial curves (that is, in case $d \geq g+r$ you take a general curve $C$, a general line bundle $L$ of degree $d$ on $C$ and a general $r+1$-dimensional vector subspace of $H^{0}(L)$ - though it's far from easy to prove even in this case). By far the strongest evidence we have in general is due to Voisin ([V1] and [V2]), who proved the full MRC for canonical curves of any genus.

Other attributes of general curves

When a curve is embedded in projective space, there are other aspects of its extrinsic geometry than the algebra of its ideal, and we should mention at least a couple of these.

One is the behavior of secant planes to $C$. In general, if $C$ is a curve in $\mathbb{P}^{r}$, a naive dimension count would lead us to expect that $C$ would have $e$-secant $n$-planes if and only if

$$
(n+1)(r-n) \geq e(r-n-1)
$$

or, equivalently, when

$$
e \geq(r-n)(e-n-1)
$$

(To go somewhat $19^{\text {th }}$ century for a moment: the variety of incident $n$ planes to $C$ will have codimension $r-n-1$ in the $(n+1)(n-r)$-dimensional Grassmannian $\mathbb{G}(n, r)$; the variety of $e$-secant planes should accordingly have codimension $e(r-n-1)$. Alternatively, it should be $(r-n)(e-n-1)$ conditions of the matrix of coordinates of $e$ points on $C$ to have rank at most $n+1$.) When this inequality is satisfied, we have a formula for the class of the locus of such planes (viewed as a subvariety of the symmetric product $C_{e}$ ), and if this class is nonzero we may indeed conclude that such secant planes to $C$ exist.

But what about the other direction? If $(n+1)(r-n)<e(r-n-1)$, can we conclude that $C$ has no $e$-secant $n$-planes? And more generally, can we say that the dimension of the variety of such planes is the expected $(n+1)(r-n)-e(r-n-1)$ ? Cotterill $([\mathbf{C}])$ and Farkas $[\mathbf{F}]$ have strong results along these lines.

Another aspect of the geometry of curves in projective space is their inflectionary points. We know something about this, thanks to statements 1a' and 1b' of Brill-Noether theory, but these leave unanswered some basic questions. For example, we'd expect a general curve in $\mathbb{P}^{r}$ to have only weight one inflectionary points. Is this the case?

\section{Linear systems on special curves}

We come now to an aspect of Brill-Noether theory that is largely unexplored: what, if anything, can we say about the geometry of linear systems on special curves? Of course it's wrong to say we don't know much about linear systems on special curves: we have Clifford's theorem, describing extremal behavior among all linear systems, and Castelnuovo's theorem, describing 
extremal behavior among birationally very ample linear systems ([ACGH $]$ p. 107 and 116). Moreover, we have theorems of Martens, Mumford and Keem about the extremal behavior of the varieties $G_{d}^{r}$ on arbitrary curves ([ACGH $]$ p. 191, 193 and 200). But what we have in mind is something quite different: we'd like to know, to what extent do the basic theorems of Brill-Noether theory continue to hold for curves $C$ that are general in low-codimension subvarieties of the moduli space $M_{g}$ ?

A little explanation is in order. Recall that the scheme $\mathcal{G}_{d}^{r}$ parametrizes triples $(C, L, V)$ consisting of a smooth curve $C$ and a $g_{d}^{r}$ on $C$. Now, the same dimension counts that lead us to guess that the dimension of $G_{d}^{r}$ on a general curve is $\rho(g, r, d)$ would lead us to expect that the dimension of $\mathcal{G}_{d}^{r}$ is

$$
\rho(g, r, d)+3 g-3=4 g-3-(r+1)(g-d+r) .
$$

Is this the case?

Another way to formulate this would be in terms of the dimension of the Hilbert scheme, or at least of those components $\mathcal{H}$ of the Hilbert scheme $\mathcal{H}_{d, g, r}$ of curves of degree $d$ and genus $g$ in $\mathbb{P}^{r}$ whose general point corresponds to a smooth, irreducible nondegenerate curve $C \subset \mathbb{P}^{r}$. An open subset of any such component is a $P G L_{r+1}$-bundle over $\mathcal{G}_{d}^{r}$; the dimension count above would lead us to conclude that the dimension of $\mathcal{H}$ is

$$
\begin{aligned}
\operatorname{dim} \mathcal{H}=\lambda(g, r, d) & =4 g-3-(r+1)(g-d+r)+(r+1)^{2}-1 \\
& =4 g-4+(r+1)(d-g+1) .
\end{aligned}
$$

Again, is this the case?

There is another way of arriving at this estimate on $\operatorname{dim} \mathcal{H}$. If $[\mathbf{C}] \in \mathcal{H}$ is a general point, we can estimate $\operatorname{dim} \mathcal{H}$ by the dimension of the Zariski tangent space to $\mathcal{H}$ at $[\mathbf{C}]$, which is given as the space of sections $H^{0}(N)$ of the normal bundle $N=N_{C / \mathbb{P}^{r}}$ of $C \subset \mathbb{P}^{r}$. If we think of the Euler characteristic $\chi\left(N_{C / \mathbb{P}^{r}}\right)$ as a first approximation to the dimension of this space, we're led to the estimate

$$
\begin{aligned}
\lambda(g, r, d) & =\operatorname{deg} N-(r-1)(g-1) \\
& =(r+1) d-(2-2 g)-(r-1)(g-1) \\
& =4 g-4+(r+1)(d-g+1) .
\end{aligned}
$$

Now, this is ridiculous, even by the extremely loose standards of this paper. It turns out to be correct in the cases $r=1$ and $r=2$, but as soon as we get to $r \geq 3$ it is wrong by an order or magnitude. For example, look at the component of the Hilbert scheme parametrizing smooth curves of type $(a, b)$ on a quadric $Q \subset \mathbb{P}^{3}$. (Such curves will actually form an open subset of the Hilbert scheme when $a$ and $b$ are both $\geq 3$.) Since $Q$ varies with 9 degrees of freedom and the linear system of such curves on a given quadric 
has dimension $(a+1)(b+1)-1$, we have

$$
\operatorname{dim} \mathcal{H}=(a+1)(b+1)+8 .
$$

On the other hand, we have

$$
\begin{aligned}
\lambda & =4 g-4+4(d-g+1) \\
& =4 d \\
& =4(a+b) .
\end{aligned}
$$

Or try curves $C \subset \mathbb{P}^{3}$ that are complete intersections of pencils of surfaces of degree $a$ : the Grassmannian of such pencils has dimension

$$
\begin{aligned}
\operatorname{dim} \mathcal{H} & =\operatorname{dim} G\left(2,\left(\begin{array}{c}
a+3 \\
3
\end{array}\right)\right) \\
& =\frac{(a+3)(a+2)(a+1)}{3}-4
\end{aligned}
$$

whereas again

$$
\lambda=4 d=4 a^{2} .
$$

Similarly, virtually all the curves we can actually write down in projective space - complete intersections, determinantal curves, and curves on rational surfaces - violate this estimate. But the observation that really points out the absurdity of the estimate in general is this: if you fix $r \geq 4$ and a reasonably large $d$, and look at the expression for $\lambda(g, r, d)$ above, you'll see that

$$
\lambda=(r+1) d-(r-3)(g-1) .
$$

But irreducible, nondegenerate curves of degree $d$ in $\mathbb{P}^{r}$ have genera ranging from 0 to Castelnuovo's bound

$$
\pi(d, r) \sim \frac{d^{2}}{2(r-1)}
$$

in other words, the value of $\lambda$ is actually negative for the majority of possible values of $g$ allowed by Castelnuovo's bound!

So, if it's absurd to expect that the dimension of the Hilbert scheme satisfies the naive estimate $\operatorname{dim} \mathcal{H}=\lambda(g, r, d)$, why are we even mentioning it? Well, here's the striking thing: while it's not even the right order of magnitude as a function of $d, g$ and $r$ in general, it does seem to hold in low codimension in $M_{g}$. In fact, evidence (or, more properly, lack of evidence to the contrary) suggests the

Conjecture: If $\mathcal{H}$ is any component of the Hilbert scheme whose general member corresponds to a smooth, irreducible, nondegenerate curve of degree $d$ in $\mathbb{P}^{r}$, and the image of the rational map $\mathcal{H} \rightarrow M_{g}$ has codimension $g-4$ or less, then

$$
\operatorname{dim} \mathcal{H}=\lambda(g, r, d)
$$


To be honest, the available evidence suggests simply the existence of a number $\beta(g)$ tending linearly to $\infty$ with $g$, such that any such component $\mathcal{H}$ whose image in $M_{g}$ has codimension $\beta \leq \beta(g)$ has the expected dimension; we use the function $g-4$ just for simplicity. What's fascinating to me is really the qualitative behavior: that the estimate $\operatorname{dim} \mathcal{H}=\lambda$ seems to hold for curves that are not "too" special, but of course fails utterly in higher codimension in $M_{g}$

Problem: Find a lower bound for the dimension of a component of the Hilbert scheme whose general point corresponds to an irreducible, nondegenerate curve of degree $d$ and genus $g$.

In particular, we don't even know if there exist rigid curves - that is, curves with no nontrivial deformations in projective space- other than rational normal curves.

\section{References}

[ACGH] Enrico Arbarello, Maurizio Cornalba, Phillip Griffiths and Joe Harris, Geometry of Algebraic Curves, Springer-Verlag 1985.

[C] Ethan Cotterill, Geometry of curves with exceptional secant planes, arXiv:0706.2049.

[BE] Edoardo Ballico and Philippe Ellia, The maximal rank conjecture for nonspecial curves in $\mathbb{P}^{n}$, Math. Z. 196 (1987), no. 3, 355-367.

[EH] David Eisenbud and Joe Harris, Irreducibility and monodromy of some families of linear series, Ann. Sci. cole Norm. Sup. (4) 20 (1987), no. 1, 65-87.

[ES] David Eisenbud and Frank Schreyer, personal communication.

[F] Gavril Farkas, Higher ramification and varieties of secant divisors on the generic curve, J. Lond. Math. Soc. (2) 78 (2008), no. 2, 418-440.

[HM] Joe Harris and Ian Morrison, Moduli of Curves, Springer-Verlag 1991.

[V2] Claire Voisin, Green's generic syzygy conjecture for curves of even genus lying on a K3 surface, J. Eur. Math. Soc. (JEMS) 4 (2002), no. 4, 363-404.

[V2] Claire Voisin, Green's canonical syzygy conjecture for generic curves of odd genus, Compos. Math. 141 (2005), no. 5, 1163-1190. 
